FOLIA

Amazónica

Revista del Instituto de Investigaciones

de la Amazonía Peruana

\title{
USO DE UN ENRAIZANTE EN LA PROPAGACIÓN VEGETATIVA DEL PLÁTANO (Musa paradisiaca L.) CLON HARTON EN CONDICIONES DE CÁMARA TÉRMICA
}

\author{
Markyori Sofí SALVADOR CARHUARUPAY ${ }^{1}$, Jessica Madeley RÍOS GUZMÁN ${ }^{1 *}$, \\ Pablo Pedro VILLEGAS PANDURO ${ }^{1}$, Fernando PÉREZ LEAL ${ }^{1}$ \\ 1 Universidad Nacional de Ucayali (UNU), Carretera Federico Basadre km 6,2, distrito de Callería, \\ provincia de Coronel Portillo, Ucayali, Perú. \\ *Correo electrónico: jessica_rios@unu.edu.pe
}

\section{RESUMEN}

El objetivo en este trabajo fue determinar el efecto de diferentes dosis del enraizante Root-Hor® en la multiplicación de hijuelos del clon de plátano Hartón (plátano bellaco) bajo condiciones de cámara térmica en la provincia de Padre Abad, Aguaytía, Perú, para lo cual, se construyó una cámara térmica y en las camas de enraizamiento, se utilizó compost como base, formando una capa de $10 \mathrm{~cm}$, seguido, se espolvoreó roca fosfórica en toda la cama y finalmente, se agregó una capa de aserrín de $40 \mathrm{~cm}$ de espesor. Se seleccionaron cormos de plantas madres sanas, con un promedio de peso de $2 \mathrm{~kg}$, al cual se le eliminó el punto de crecimiento central haciendo un corte en cono de $4 \mathrm{~cm}$ de profundidad en cada cormo. Fueron desinfectados con una solución de hipoclorito de sodio $5 \%$ por 3 minutos, enseguida fueron tratados con el enraizante Root-Hor $\AA$ de acuerdo a las dosis estudiadas $(0 \mathrm{ml} / 100 \mathrm{~L}, 50 \mathrm{~m} \mathrm{l} / 100 \mathrm{~L}, 100 \mathrm{ml} / 100 \mathrm{~L}$, $150 \mathrm{ml} / 100 \mathrm{~L}$ y $200 \mathrm{ml} / 100 \mathrm{~L}$ ), finalmente fueron colocados en las camas de enraizamiento, dentro de la cámara térmica, demostrándose que la aplicación de 150 ml/100 L de Root-Hor@ logró 8,2 hijuelos/cormo, 330,4 g de peso/hijuelo, 118,2 cm de altura de hijuelo, 4 cm de diámetro de hijuelo, 20,4 raíces/hijuelo y $25 \mathrm{~cm}$ de longitud de raíces, superando a las otras aplicaciones de Root-Hor $₫$ y al testigo, mostrando eficiencia en la propagación vegetativa del clon Hartón en condiciones de cámara térmica.

PALABRAS CLAVE: ácido alfa naftalenacético (AAN), ácido índolbutírico (AI), cormos, enraizamiento, macollamiento. 


\title{
USE OF A ROOTING AGENT IN THE VEGETATIVE PROPAGATION OF BANANA (Musa paradisiaca L.) HARTON CLONE UNDER THERMAL CHAMBER CONDITIONS
}

\begin{abstract}
The objective in this work was to determine the effect of different doses of the rooting agent Root-Hor $\AA$ in the multiplication of suckers of the Harton clone (bellaco plantain) under thermal camera conditions in the province of Padre Abad, Aguaytía, Peru. A thermal chamber $3 \mathrm{~m}$ wide $\mathrm{x} 5 \mathrm{~m}$ long and $1.8 \mathrm{~m}$ high was built, lined with transparent plastic, and in the rooting beds, compost was used as a base, forming a layer of $10 \mathrm{~cm}$, followed by phosphate rock was sprinkled all over the bed and finally a $40 \mathrm{~cm}$ thick layer of sawdust was added. Corms of healthy mother plants were selected, with an average weight of $2 \mathrm{~kg}$, from which the central growth point was eliminated by making a cone cut $4 \mathrm{~cm}$ deep in each corm, which were disinfected with a solution of 5\% sodium hypochlorite for 3 minutes, then they were treated with the Root-Hor@ rooting agent according to the doses studied $(0 \mathrm{ml} / 100 \mathrm{~L}, 50 \mathrm{ml} / 100 \mathrm{~L}, 100 \mathrm{ml} / 100 \mathrm{~L}, 150 \mathrm{ml} / 100 \mathrm{~L}$ and $200 \mathrm{ml} / 100 \mathrm{~L}$ ), finally they were placed in the rooting beds, inside the thermal chamber, showing that the application of $150 \mathrm{ml} / 100 \mathrm{~L}$ of Root-Hor $₫$ achieved 8.2 suckers / corm, $330.4 \mathrm{~g}$ of weight / suckling, $118.2 \mathrm{~cm}$ of sucker height , 4 $\mathrm{cm}$ diameter of sucker, 20.4 roots / sucker and $25 \mathrm{~cm}$ of root length, surpassing the other applications of Root-Hor $®$ and the control, showing efficiency in the vegetative propagation of the Hartón clone under thermal chamber conditions.

KEYWORDS: alpha naphthaleneacetic acid (ANA), indolbutyric acid (IA), corms, rooting, tillering.
\end{abstract}




\section{INTRODUCCIÓN}

La familia de las musáceas muestra un rol importante en la seguridad alimentaria, siendo los países con mayor consumo per cápita: Ecuador, Cuba, República Dominicana y Colombia, con 320, $210,200,160 \mathrm{~g} /$ día por persona, respectivamente (MADR, 2006).

En América Latina y el Caribe, las variedades tipo French (Dominico), False Horn (Dominico Hartón) y Horn (Hartón) corresponden a los plátanos tipo Plantain más cultivados, siendo el False Horn y Horn muy comercializados y susceptibles a sigatoka negra (Arcila, 2002). Cayón et at. (2000) afirman que, en Colombia, las variaciones de las condiciones ambientales influyen en la calidad de los frutos de plátano Dominico Hartón, principalmente, el estrés hídrico, en la etapa del desarrollo del racimo.

La propagación del plátano por medio de cormos, rizomas o bulbos, se dividen de 4 a 8 partes, los que pueden generar 500 nuevos retoños de un solo cormo en un lapso de 8 meses. Esto se da por la pérdida de la dominancia apical, la cual activa las yemas laterales, generando mayor número de hijuelos por cormo (Herrera \& Colonia, 2011).

En ese sentido, FONTAGRO (2010) aplicando la técnica de reproducción acelerada de semillas a cormos de 1 y $2 \mathrm{~kg}$ de la variedad Dominico Hartón y utilizando aserrín esterilizado como sustrato de siembra, dentro de cámara térmica, obtuvo hasta 90 brotes $/ \mathrm{m}^{2}$ por mes, superando a los 35 brotes $/ \mathrm{m}^{2}$ por mes que se producen en condiciones ambientales naturales.

Los bioestimulantes aumentan expresiones metabólicas y/o fisiológicas de la planta, tales como el desarrollo de raíces, frutos, etc., incrementando la fotosíntesis y reduciendo los daños causados por estrés, fortaleciendo la defensa natural de las plantas, mejorando así el estado nutricional de la planta, y el equilibrio hormonal, con la síntesis de auxinas, gibelinas y citoquininas (Alban, 2014).

Actualmente hay una tendencia de reemplazar el uso de los reguladores de crecimiento sintéticos por productos bioactivos de origen biológico, buscando reducir los costos de producción (Díaz et al., 2004). Entre los bioestimulantes comerciales que se están utilizado se encuentra Biobras (análogo de brasinoesteroides), el Bioestan y Liplant (derivados del humus de lombriz y el vermicompost), el Fitomas-E (derivado de la caña de azúcar) y el Rizobac (de origen rizobacteriano) (Rodríguez et al., 1999; Héctor et al., 2002; Pérez, 2007).

El Bioestimulante Root-Hor $®$ está constituido por: ácido alfa-naftalenacético $(0,40 \%)$, ácido 3-indolbutírico $(0,10 \%)$, ácidos nucleicos $(0,10 \%)$, sulfato de zinc $(0,40 \%)$ y solución nutritiva (95,40\%); que favorece la acción de las auxinas en forma armónica en los tejidos celulares y en la planta. Estimulando la formación de raíces, especialmente en estacas, acodos y esquejes de diversos cultivos (Grupo Andina, 2014)

En ese contexto, el objetivo en este trabajo fue determinar el efecto de cuatro dosis del enraizante Root-Hor ${ }^{\circledR}$ en la propagación vegetativa del plátano clon Harton, bajo condiciones de cámara térmica, en la provincia de Padre Abad.

\section{MATERIAL Y MÉTODOS}

La investigación se desarrolló en el vivero agrícola de la Facultad de Ciencias Agropecuarias de la Universidad Nacional de Ucayali - Sede Aguaytia, distrito de Padre Abad, ubicada a: Latitud: $08^{\circ} 25^{\prime} 76^{\prime \prime}$ S.; Longitud: $74^{\circ} 53^{\prime} 00^{\prime \prime}$ O.; Altitud: $284 \mathrm{msnm}$.

\section{CONSTRUCCIÓN DE LA CÁMARA TÉRMICA}

Se limpió un área de $18 \mathrm{~m}^{2}$, para establecer la cámara térmica, de $3 \mathrm{~m}$ de ancho y $5 \mathrm{~m}$ de largo, 
y una altura de 1,8 m. Para su construcción se utilizó postes de madera y bambú de la zona, forrado con plástico transparente.

A las camas de enraizamiento se les aplicó una capa de compost de $10 \mathrm{~cm}$ de espesor como base, se le espolvoreó roca fosfórica y finalmente, se agregó una capa de aserrín de madera blanca y fresca, de $40 \mathrm{~cm}$ de espesor.

\section{RECOLECCIÓN Y SELECCIÓN DE CORMOS}

Se obtuvieron cormos de $2 \mathrm{~kg}$ peso, provenientes de plantas madres sanas, con excelente producción de racimos, procediendo a quitarles todas las hojas. Se eliminó el punto de crecimiento central, haciendo un corte en cono de $4 \mathrm{~cm}$ de profundidad en cada cormo y se realizó un corte en cruz de $2 \mathrm{~cm}$ de profundidad.

Los cormos se sumergieron en un recipiente de 20 litros de capacidad, conteniendo $50 \mathrm{~cm}^{3}$ de hipoclorito de sodio al 5\% y Oncol (Benfuracarb) $40 \mathrm{ml} / 20 \mathrm{~L}$, durante 3 minutos para su desinfección.

\section{APLICACIÓN DE ENRAIZANTES EN LOS CORMOS}

Los cormos desinfectados, se sumergieron en una bandeja de plástico, por un espacio de media hora, aplicando las dosis de enraizante $(\mathrm{T} 1=$ sin enraizante; T2 $=50 \mathrm{ml} / 100 \mathrm{ml}$ de RootHor®; T3 = 100 ml/100 ml de Root-Hor®; T4 = $150 \mathrm{ml} / 100 \mathrm{ml}$ de Root-Hor®; T5 = 200 ml/100 $\mathrm{ml}$ de Root-Hor $\AA$ ). Posteriormente, se extrajeron los cormos y se dejaron escurrir por 10 minutos, para luego ser instalados en las camas de enraizamiento, dentro de la cámara térmica.

\section{INSTALACIÓN DE LOS CORMOS Y MANEJO DEL RIEGO}

En las camas de enraizamiento, se instalaron 20 cormos por $1 \mathrm{~m}^{2}$, con una separación de $20 \mathrm{~cm}$ entre ellos. Los cormos se cubrieron con una capa de $5 \mathrm{~cm}$ de aserrín descompuesto, seguidamente, se utilizaron regaderas para humedecer las camas.

Se realizaron riegos diarios, por la mañana, para mantener la humedad en capacidad de campo en las camas de enraizamiento.

\section{CONTROL DE LA TEMPERATURA}

La temperatura registrada dentro de la cámara térmica estuvo en un rango de $32{ }^{\circ} \mathrm{C}$ a $55^{\circ} \mathrm{C}$, cuando la temperatura superaba los rangos favorables, las puertas de la cámara fueron abiertas para favorecer la circulación de aire.

Las variables número de hijuelos por cormo, peso, altura y diámetro de hijuelos, número de hojas y raíces por hijuelo y longitud de raíces fueron evaluadas a los 45 días, finalizando el experimento.

\section{DISEÑO ESTADÍSTICO}

El estudio fue conducido mediante un Diseño Completamente al Azar (DCA), con 5 tratamientos, 4 repeticiones y 10 cormos por unidad experimental

\section{ANÁLISIS DE DATOS}

Los datos fueron tabulados en el software Excel (2019), en seguida se realizó la prueba de normalidad de datos de Shapiro-Wilk y homogeneidad de varianza. Luego de hacer la normalización, los datos fueron analizados mediante la prueba de $\mathrm{F}$ a $5 \%$ de probabilidad. Las medias fueron sometidas a pruebas de comparaciones múltiples de Tukey $(\alpha=0,05)$. Todos los análisis estadísticos fueron realizados por medio del programa estadístico SAS® (Statistical Analysis System) versión 8.1. 
Tabla 1. Número, peso, altura y diámetro de hijuelos por cormo de la variedad Hartón con aplicación de enraizante Root-Hor ${ }^{\circledR}$, en cámara térmica.

\begin{tabular}{cccccc}
\hline Trat. & Descripción & $\begin{array}{c}\text { No de hijuelos/ } \\
\text { cormo }\end{array}$ & $\begin{array}{c}\text { Peso de hijuelos } \\
\mathbf{( g )}\end{array}$ & $\begin{array}{c}\text { Altura de } \\
\text { hijuelos }(\mathbf{c m})\end{array}$ & $\begin{array}{c}\text { Diámetro de } \\
\text { hijuelos }(\mathbf{c m})\end{array}$ \\
\hline T1 & sin enraizante & $5,6 \mathrm{c}$ & $169,2 \mathrm{~d}$ & $78,6 \mathrm{e}$ & $2 \mathrm{c}$ \\
T2 & $50 \mathrm{ml} / 100 \mathrm{~L}$ de Root-Hor & $5,6 \mathrm{c}$ & $239 \mathrm{c}$ & $86,8 \mathrm{~d}$ & $2 \mathrm{c}$ \\
T3 & $100 \mathrm{ml} / 100 \mathrm{~L}$ de Root-Hor ${ }^{\circledR}$ & $6,6 \mathrm{~b}$ & $266 \mathrm{~b}$ & $94,4 \mathrm{c}$ & $3 \mathrm{~b}$ \\
T4 & $150 \mathrm{ml} / 100 \mathrm{~L}$ de Root-Hor ${ }^{\circledR}$ & $8,2 \mathrm{a}$ & $330,4 \mathrm{a}$ & $118,2 \mathrm{a}$ & $4 \mathrm{a}$ \\
T5 & $200 \mathrm{ml} / 100 \mathrm{~L}$ de Root-Hor ${ }^{\circledR}$ & $7 \mathrm{~b}$ & $236,8 \mathrm{c}$ & $104,6 \mathrm{~b}$ & $3 \mathrm{~b}$ \\
\hline
\end{tabular}

Letras iguales no presentan diferencias significativas. Tukey $p \leq 0,05$

\section{RESULTADOS Y DISCUSIÓN}

\section{NÚMERO, PESO, ALTURA Y DIÁMETRO DE} HIJUELOS POR CORMO

El tratamiento T4, logró los mayores valores para el número, peso, altura y diámetro de hijuelos por cormo, siendo, además, los tratamientos con aplicación de Root-Hor ${ }^{\circledR}$ superiores al tratamiento sin aplicación de enraizador, el cual logró menores valores para todas las variables evaluadas (Tabla 1). Resultados similares fueron reportados por Ozambela (2017) quien afirma que el enraizante Root-Hor® generó mayor número de hijuelos brotados y mayor número de hijuelos cosechados a los 60, 90 y 120 días, concordando con Álvarez et al. (2013), quienes lograron hasta 90 brotes $\mathrm{m}^{2}$ por mes de la variedad Dominico Hartón, dentro de la cámara térmica, en comparación con los 35 brotes por $\mathrm{m}^{2}$ por mes en condiciones convencionales, siendo estos resultados inferiores a los obtenidos con la aplicación del tratamiento T4, en el cual se obtuvieron 160 plantones por $\mathrm{m}^{2}$.

Cedeño-García et al. (2016), observaron mayor tasa de multiplicación a $40 \mathrm{mg} \mathrm{L}-1$ de bencilaminopurina (BAP), con 47,27 hijuelos/ cormo, y a 80 mg L-1 de BAP, con 45,32 hijuelos/ cormo del banano cv. Williams en condiciones de cámara térmica, concordando con Meza-Tuarez
(2013) el cual demostrando que con $60 \mathrm{mg} \mathrm{L-1}$ BAP y una intensidad lumínica de $50 \%$, generó el mayor número de rebrotes totales y mayor rentabilidad, en plátano variedad Dominique.

Ayunque-Briceño \& Inga-Lobo (2019) demostraron que la variedad de banano Valery con una dosis de $0,8 \mathrm{~g}$ de ácido Giberélico, generó en promedio 38,67 hijuelos, seguido de la dosis de 0,4 g de ácido Giberélico, con un promedio de 35,67 hijuelos, en condiciones de cámara térmica, siendo estos resultados inferiores a los obtenidos con la aplicación del tratamiento T4, en el cual generó 160 hijuelos por $\mathrm{m}^{2}$.

Los resultados obtenidos en la presente investigación, concuerdan con lo indicado por Farabee (2007), al indicar que las auxinas influyen en el crecimiento de los órganos vegetales, estimulando la elongación o alargamiento de ciertas células, asimismo, Grupo Andina (2014), afirma que Root-Hor $®$ es un producto que penetra en los tejidos celulares elevando las concentraciones de auxinas, estimulando el desarrollo radicular.

\section{NÚMERO DE HOJAS, NÚMERO Y LONGITUD} DE RAÍCES

Latabla 2 muestra que, para el número de hojas por hijuelos, no se observan diferencias significativas entre los tratamientos estudiados, sin embargo, 
Tabla 2: Número de hojas, número y longitud de raíces de la variedad Hartón con aplicación de enraizante Root-Hor ${ }^{\circledR}$, en cámara térmica.

\begin{tabular}{ccccc}
\hline Trat. & Descripción & No de hojas/hijuelo & No de raíces/hijuelo & $\begin{array}{c}\text { Longitud de raíces } \\
\text { (cm) }\end{array}$ \\
\hline T1 & sin enraizante & $3 \mathrm{a}$ & $13,6 \mathrm{~d}$ & $16,8 \mathrm{~d}$ \\
T2 & $50 \mathrm{ml} / 100 \mathrm{~L}$ de Root-Hor ${ }^{\circledR}$ & $3 \mathrm{a}$ & $15,4 \mathrm{c}$ & $18,2 \mathrm{~d}$ \\
T3 & $100 \mathrm{ml} / 100 \mathrm{~L}$ de Root-Hor & $3 \mathrm{a}$ & $16,6 \mathrm{~b}$ & $20,2 \mathrm{c}$ \\
T4 & $150 \mathrm{ml} / 100 \mathrm{~L}$ de Root-Hor ${ }^{\circledR}$ & $3,2 \mathrm{a}$ & $20,4 \mathrm{a}$ & $25 \mathrm{a}$ \\
T5 & $200 \mathrm{ml} / 100 \mathrm{~L}$ de Root-Hor ${ }^{\circledR}$ & $2,8 \mathrm{a}$ & $16,2 \mathrm{bc}$ & $22,2 \mathrm{~b}$ \\
\hline
\end{tabular}

Letras iguales no presentan diferencias significativas. Tukey $p \leq 0,05$

para el número y longitud de raíces por hijuelo, el tratamiento T4, logró los mayores valores, siendo, además, los tratamientos con aplicación de RootHor® superiores al tratamiento sin aplicación de enraizador, el cual logró menores valores para las variables evaluadas.

Cutire \& Bautista (2014) indica que la dosis de $3,75 \mathrm{ml} \mathrm{L}^{-1}$ de ácido indolbutírico (AI) generó una mayor altura de hijuelo $(31 \mathrm{~cm})$, siendo la dosis de 2,5 $\mathrm{ml} \mathrm{L}^{-1}$ la que generó mayor peso fresco y seco de raicillas por hijuelo $(10,4 \mathrm{~g}$ y 5,44 g, respectivamente) en plátano var. Bellaco. Asimismo, Quichimbo (2014) obtuvo mayor material vegetativo con raíces activas en el tratamiento con media cepa, 1 yemas y Kelpak $10 \mathrm{ml}(56,4 \%)$ en el banano variedad William, superando al sistema de propagación tradicional. Sin embargo, Canchignia-Martínez et al. (2008) que al aplicar $30 \mathrm{mg} \mathrm{L}^{-1}$ de BAP, lograron valores de 2,36 brotes para las variedades Banano Valery y Orito y del Plátano Barraganete, demostrándose el efecto estimulador de las auxinas y citocininas, en la propagación vegetativa de diferentes variedades de plátanos y bananos.

Cutire-Espinoza \& Astorga-Neira (2014) afirman que el AI aplicado a una dosis de $3,75 \mathrm{ml} \mathrm{L}^{-1}$, estimula el crecimiento de la parte aérea y a dosis $2,5 \mathrm{ml} \mathrm{L}^{-1}$ estimula el enraizamiento, favoreciendo la propagación vegetativa por división de cormos del plátano variedad Bellaco en invernadero. De similar manera, Quispe-Huamán (2017) comparó el efecto de tres dosis del bioestimulante Rooter (a base de AI), en cormos de plátano variedad Bellaco, en invernadero, demostrando que la dosis de 3,75 $\mathrm{ml} \mathrm{L}^{-1}$ de Rooter logró mayor altura de planta, mayor número de hojas y mostró la mejor rentabilidad económica, lo cual coincide con el efecto del Root-Hor®, el cual contiene AI en su constitución de reguladores de crecimiento.

Ospina-Flores \& Rubiano-Moncada (2019) demostraron que el Aquaclean ACF (-) SF(+), y el ácido fosfórico (AF), generaron mayor emisión de raíces, con mayor longitud y diámetro, así como un mejor desarrollo de altura, diámetro del pseudotallo y producción de hojas de las plántulas de plátano Dominico-Hartón. Finalmente, Pizarro-Mena (2019) demostró que el extracto de lentejas y Ryzup 40 (compuesto sintético) presentaron los más altos porcentajes de raíces sanas (60\%) en el banano clon Williams, siendo estas investigaciones coincidentes con el presente estudio, en el uso de compuestos bioestimulantes a base de auxinas para generar 
mejores promedios de desarrollo vegetativo y radicular de los hijuelos, a partir de los cormos del clon Hartón, en comparación a los sistemas de propagación vegetativa tradicional.

\section{CONCLUSIONES}

La aplicación de 150 ml/100 L de Root-Hor®, generó los mejores valores de número, peso, altura y diámetro de hijuelos por cormo, así como el mayor número y longitud de raíces por hijuelo del plátano variedad Hartón en cámara de térmica, siendo el tratamiento sin aplicación de enraizador el que presentó los menores valores de desarrollo vegetativo de hijuelos por cormo.

\section{REFERENCIAS BIBLIOGRÁFICAS}

Álvarez, E.; Ceballos, G.; Gañán, L.; Rodríguez, D.; González, S.; Pantoja, A. 2013. Producción de material de 'siembra' limpio en el manejo de las enfermedades limitantes del plátano. Centro Internacional de Agricultura Tropical (CIAT). Cali - Colombia 16pp.

Alban, E. 2014. Evaluación de la eficacia de citoquinina (Cytokin) y un inductor carbónico (Carburos) en tres dosis y en dos épocas en el rendimiento de banano de exportación, en una plantación en producción variedad enana, Canton Quinide de la provincia de Esmeralda. Riobamba, Ecuador. Tesis de pregrado, Escuela Superior de Politécnica de Chimborazo, Riobamba - Ecuador. 112pp.

Arcila, P. 2002. Aceptabilidad por el consumidor de los plátanos África 1 y FHIA 21 en el departamento del Quindio, Colombia. En: Corrales, 0; Knight, S.; Madrigal, M. (Eds.) Memorias. $15^{\circ}$ Reunión Internacional Acorbat, Cartagena, 2002. p. 578-581.
Ayuque-Briceño, L.V.; Inga-Lobo, J.R. 2019. Aplicación de bioregulador en la propagación vegetativa en variedades de banano (Musa paradisiaca L.) en cámara térmica - Distrito de San Ramón - Chanchamayo. Tesis de pregrado, Universidad Nacional Daniel Alcides Carrión, La Merced - Perú. 120pp.

Canchignia-Martínez, H.F.; Espinoza-Roca, D.E.; Benavides-Velásquez, G.R.; Saucedo-Aguiar, S.G.; Carranza-Patiño, M.S.; Cevallos-Falquez, O.F. 2008. Propagación vegetativa de plátano y banano con la aplicación de benzilaminopurina (6-BAP) y ácido indolacético (AIA). Ciencia y Tecnología, 1: 11-15. 2008.

Cayón, D.; Giraldo, G.G.A.; Aecila, M.I. 2000. Postcosecha y agroindustria del plátano en el eje cafetero de Colombia. Corporación Colombiana de Investigación Agropecuaria (Corpoica). Corporación Colombiana de Investigación Agropecuaria (Corpoica), Armenia - Colombia. 246pp.

Cedeño-García, G.; Soplín-Villacorta, H.; HelfgottLerner, S.; Cedeño-García, G.; SotomayorHerrera, I. 2016. Aplicación de biorreguladores para la macro-propagación del banano cv. Williams en cámara térmica. Agronomía Mesoamericana, 27(2): 397-408. DOI: https:// doi.org/10.15517/am.v27i2.24390

Cutire-Espinoza, G.; Astorga-Neira, J.B. 2014. Efecto del ácido indol butírico en la propagación de plátano var. Bellaco (Musa balbisiana Colla) en Echarate, La Convención. Tesis de pre-grado, Universidad Nacional del Altiplano, Puno, Perú. 86pp.

Díaz, B.; Héctor, E.; Torres, A.; Cabañas, M.; Garcés, N.; Izquierdo, H.; Núñez, M.; Iglesias, R. 2004. Empleo de productos bioactivos cubanos como sustitutos de los reguladores del crecimiento en la propagación del plátano (AAB) en fase de establecimiento in vitro. Alimentaria: Revista de Tecnología e Higiene de los Alimentos, 359:103-108. 
Farabee, M.J. 2007. Plant hormones, nutrition and transport. (https://www2.estrellamoun tain.edu/faculty/farabee/biobk/BioBook PLANTHORM.html). Acceso: 26/11/2020.

FONTAGRO (Fondo Regional de Tecnología Agropecuaria). 2010. Fortalecimiento de cadenas de valor de plátano: Innovaciones tecnológicas para reducir agroquímicos. (https://www.fontagro.org/wp-content/ uploads /2006/01/0605-06-Informe-Te\% CC\%81cnicoFinal.pdf). Acceso: 03/11/2020.

Grupo Andina. 2014. Ficha técnica de Root Hor. (http://www.grupoandina.com.pe/media/ uploads/ficha_tecnica/roothor-_ficha_ tecnica_pdf.pdf). Acceso: 03/11/2020.

Héctor, E.; Díaz, B.; Torres, A.; Garcés, N.; Huelva, R.; Roque, A.; Godoy, L.; Lsidrón, M.; Tirado, A.; Cabañas, M.; Cremé, Y.; Díaz, A.; Proenza, R. 2002. Efecto del Liplant y el Biostan en la propagación in vitro del plátano macho (Musa sp. AAB). En: 9no Seminario Científico Internacional AGROTROP 2002. p. 1-6.

Herrera Rojas, M.; Colonia Coral, L. 2011. Manejo integrado del cultivo de plátano. Universidad Nacional Agraria La Molina (UNALM), La Merced, Junín. 33pp.

Meza-Tuarez, J.E. 2013. Propagación vegetativa de plátano Dominique (Musa paradisiaca) bajo dos porcentajes de sombra con la aplicación de cuatro dosis de Benzilaminopurina (BAP) en el cantón el empalme provincia del Guayas. Tesis de pre-grado. Universidad Técnica de Cotopaxi, Cotopaxi, Ecuador. 81pp.

Ospina-Flores, J.A.; Rubiano-Moncada, J. 2019. Evaluación de bio-estimulantes en la propagación intensiva de semilla plátano Dominico Hartón en almácigo bajo cubierta plástica. Tesis de pre-grado. Universidad Nacional Abierta y a Distancia - UNAD, Risaralda, Colombia. 72pp.

Ozambela Torres, L. 2017. Efecto de tres enraízantes sintéticos en la producción de hijuelos de plátano (Musa paradisiaca L.) bajo condiciones de la cámara térmica. Tesis de pre-grado. Universidad Nacional Agraria de la Selva, Tingo María, Perú. 96pp.

Pérez, J. 2007. Efecto de algunos reguladores del crecimiento y el Fitomás- $E$ en la micro propagación de Musa sp. variedad FHIA-18 $(A A A B)$. Centro de Estudios de Biotecnología Vegetal, Universidad de Granma, Cuba. 13pp.

Pizarro-Mena, G. L. 2019. Efectos de los enraizadores en la velocidad del retorno en banano (Musa $x$ paradisiaca) clon Williams. Tesis de pre-grado. Universidad Técnica de Machala, Machala, Ecuador. 66pp.

Quichimbo Ochoa, J.A. 2014. Evaluación del enraizamiento a partir de la aplicación de un biorregulador de crecimiento en yemas de banano (Musa sp.) con la variedad William. Tesis de pre-grado. Universidad Técnica de Machala, Machala, Ecuador. 55pp.

Quispe-Huamán, H. 2017. Diferentes dosis del ácido Indol Butírico en la propagación de plátano variedad Bellaco (Musa balbisiana Colla) en condiciones de invernadero, Pacobamba - Apurimac. Tesis de pre-grado. Universidad Tecnológica de los Andes, Abancay, Perú. 112pp.

Rodríguez, V.; Rodríguez, O.; Bravo, P. 1999. Índice de balance de nutrimentos DRIS (IBN-DRIS) para la predicción del rendimiento del plátano (Musa AAB subgrupo plátano cv. Hartón). Revista de la Facultad de Agronomía, 16(5): 488-494.

Recibido: 29 de diciembre de 2020 Aceptado para publicación: 30 de junio de 2021 